\title{
Adaptação da "Critical Time Intervention" para o contexto brasileiro e sua implementação junto a usuários dos Centros de Atenção Psicossocial do município do Rio de Janeiro
}

\author{
Adaptation of Critical Time Intervention for use in Brazil \\ and its implementation among users of Psychosocial Service Centers \\ (CAPS) in the municipality of Rio de Janeiro
}

\author{
Maria Tavares Cavalcanti ${ }^{1}$ \\ Maria Cecília de Araújo Carvalho ${ }^{2}$ \\ Elie Valência ${ }^{3}$ \\ Catarina Magalhães Dahl ${ }^{4}$ \\ Flávia Mitkiewicz de Souza ${ }^{1}$
}

${ }^{1}$ Laboratório de Avaliação de Serviços e Qualidade de vida em Saúde Mental, Instituto de Psiquiatria, Universidade Federal do Rio de Janeiro. Av. Venceslau Brás 71/Fundos, Botafogo.

22290-140 Rio de Janeiro RJ.ipub@ipub.ufrj.br ${ }^{2}$ Escola Politecnica de Saúde Joaquim Venancio, Fiocruz

${ }^{3}$ Universidade de Columbia

${ }^{4}$ Universidade Estadual de

Campinas (UNICAMP)
Abstract Brazilian Psychiatric Reform proposes a mental healthcare model based on the implementation of a community-based service network, in which Psychosocial Service Centers (CAPS) play a fundamental role. The report presents the results of a pilot study which aimed to adapt Critical Time Intervention to the Brazilian context, and to test its feasibility to provide it to persons with schizophrenic spectrum disorders who are enrolled in CAPS of Rio de Janeiro. Methods: The research design included three inter-related phases. Phase one consisted in carrying out qualitative and quantitative field work. This phase included mapping out the socio-demographic, clinical and service utilization data of CAPS users, as well as assessing the mental health needs of participants in the study. The second phase consisted in translation of the CTI clinical manual to include the adaptations made for use in Brazil, which were based on data collected in the first phase, as well as training individuals with moderate education as CTI intervention workers. The third phase consisted of pilot implementation of the adapted intervention among a group of individuals with schizophrenia spectrum disorders enrolled in CAPS, but with difficulties in being included in treatment.

Key words CAPS, Continuity of care, Critical time intervention, Severe and persistent mental illness
Resumo A Reforma Psiquiátrica Brasileira propõe um modelo de atenção baseado na implantação de uma rede comunitária no qual os Centros de Atenção Psicossocial (CAPS) detêm papel fundamental. Neste artigo são apresentados os resultados do estudo piloto que visou adaptar para o contexto brasileiro a "Critical Time Intervention" (CTI) e testar sua viabilidade com pessoas com transtornos do espetro esquizofrênico em tratamento nos CAPS do município do Rio de Janeiro. O desenho da pesquisa incluiu três fases inter-relacionadas. A primeira consistiu em trabalho de campo qualitativo e quantitativo. Esta fase incluiu o mapeamento das características sociodemográficas, clínicas e de utilização de serviços dos usuários dos CAPS, bem como conhecer as necessidades concretas dos participantes do estudo. A segunda fase consistiu na tradução do manual clinico da CTI para incluir as adaptações feitas para o seu uso no Brasil, oriundas dos dados coletados na primeira fase, bem como o treinamento de profissionais de nível médio para atuarem como agenciadores da CTI. A terceira fase consistiu na implementação da intervenção adaptada em um grupo de pacientes com transtornos do espectro esquizofrênicos matriculados nos CAPS, mas com dificuldade de se inserir no tratamento.

Palavras-chave CAPS, Continuidade de cuidados, Critical Time Intervention, Transtornos mentais severos e persistentes 


\section{Introdução}

A Reforma Psiquiátrica Brasileira, impulsionada a partir da década de 1970, propõe um modelo de atenção que conjuga dois processos complementares: por um lado trabalha-se para a redução de leitos psiquiátricos e por outro, incentivase a implantação de uma rede comunitária de serviços de atenção psicossocial. Os CAPS são serviços de base territorial com papel fundamental na organização da rede de saúde mental de sua área de abrangência que têm como missão o acompanhamento dos pacientes com transtornos mentais severos e persistentes ${ }^{1,2}$.

Um modelo de organização de serviços desenvolvido pela Organização Mundial de Saúde identifica os componentes essenciais que devem compor os sistemas de saúde mental. Deve-se construir uma rede integrada de cuidados, baseada na atenção primária com o suporte de serviços comunitários de saúde mental e hospitais gerais, parcerias com serviços e setores relacionados ao cuidado informal, além da participação ativa do usuário na condução de sua saúde. Este modelo propicia a redução do estigma e melhora o acesso ao cuidado ${ }^{3}$. Na base do conceito de acesso está a premissa de que os cuidados essenciais em saúde devem estar disponíveis para a população. O acesso indica o grau de facilidade com que as pessoas obtêm cuidados de saúde. Donabedian prefere o termo acessibilidade, referindo-se às características dos serviços e dos recursos de saúde que facilitam ou limitam sua utilização, enquanto Frenk privilegia a acessibilidade na procura e entrada no serviço, caracterizando-a como a relação entre os obstáculos enfrentados pela população na procura e na obtenção de cuidados de saúde e a sua capacidade de enfrentá-los ${ }^{4}$.

$\mathrm{O}$ acesso à rede de serviços de saúde se configura como uma dimensão da continuidade do cuidado, que para se efetivar requer a existência de uma rede integrada de serviços que viabilize a operacionalização de um trabalho coordenado, voltado para o plano terapêutico de cada paciente e que incorpore as esferas da vida cotidiana, como suporte social, moradia, trabalho e lazer ${ }^{5}$.

A continuidade do cuidado deve ser pensada na atenção a todo usuário do sistema de saúde, mas é um elemento que se torna mais expressivo da qualidade quando se trata do cuidado ofertado aos pacientes que utilizam diferentes serviços ao longo da vida, tais como os portadores de transtornos mentais severos e persistentes. Esta continuidade é o resultado da combinação de fatores como acesso adequado ao cuidado, habilidades comuns dos profissionais, bom fluxo de informação, entendimento entre profissionais e organizações e boa coordenação entre profissionais para manter a consistência da atenção ${ }^{6}$.

A origem da discussão teórica e conceitual sobre continuidade de cuidados remete aos anos $1960^{7}$. Diferentes definições de continuidade foram reunidas de forma esquemática por Johnson, Bass e Windle enfatizam o aspecto temporal ou longitudinal da continuidade: a continuidade é uma relação entre cuidado passado e presente, de acordo com as necessidades terapêuticas do paciente. Para Test, é fundamental que durante todo o tratamento o paciente possa ter acesso ao cuidado que necessita, podendo vir a ocorrer descontinuidade, com abandono do tratamento, quando algum elemento essencial do cuidado não estiver presente, ou quando existirem falhas na coordenação ou comunicação. Bachrach define continuidade de cuidados como um processo que envolve o movimento ininterrupto de pacientes entre os diversos elementos do sistema de prestação de cuidados ${ }^{8}$.

Para Bachrach, a continuidade do tratamento do paciente com transtornos mentais severos e persistentes depende da facilidade de acesso aos serviços. São características importantes para sua efetivação o bom acolhimento das equipes, com condutas flexíveis, pois estes pacientes podem ter dificuldades de apreender as regras para circulação no sistema de cuidados, disponibilidade para a prestação do cuidado por um longo tempo, em locais que sejam facilmente alcançados. A noção de continuidade de cuidados implica também que os serviços sejam abrangentes (o corresponde à dimensão da integralidade) indicando existência de serviços para intervenção em crises; de residências terapêuticas; de diferentes dispositivos de tratamento; oportunidades de lazer e redes de suporte social; e oferta de transporte para o acesso físico aos serviços oferecidos ${ }^{9}$.

Duas características distinguem a continuidade do cuidado de outros atributos do cuidado em saúde: o cuidado de um indivíduo e o cuidado ao longo do tempo. Ambas precisam estar presentes para que exista continuidade, embora sua presença isolada não seja suficiente para constituir continuidade do cuidado. Foram identificados três tipos de continuidade: informacional, de gerenciamento e relacional. A continuidade informacional é o fio que liga os provedores de cuidados. A continuidade da gestão é particularmente importante nas enfermidades crônicas e complexas que dependem do entendimento en- 
tre diferentes profissionais. Um aspecto importante da continuidade da gestão é a flexibilidade em adaptar o cuidado a mudanças, de acordo com as necessidades e circunstâncias específicas dos pacientes A continuidade relacional liga não somente o passado e o presente, como também promove uma ligação com o cuidado futuro ${ }^{10}$.

Estudos mostram que a boa continuidade de cuidados associa-se à melhor qualidade de vida, melhor funcionamento social, menor gravidade de sintomas e maior satisfação com o serviço ${ }^{11}$.

No final dos anos 1970 surgiram dois modelos de acompanhamento de pacientes graves na comunidade, desenhados especificamente para melhorar a continuidade de cuidados: Case Management (Gerenciamento de Casos) e Program for Assertive Community Treatment - PACT (Programa de Tratamento Assertivo na Comunidade). No case management um profissional é responsável pelo gerenciamento dos cuidados de determinado paciente na comunidade. Tal agente (case manager) deve promover o acesso aos serviços (psiquiátricos, clínicos, sociais) por ações de coordenação e integração da rede de saúde comunitária.

O outro modelo, Program for Assertive Community Treatment - PACT, caracteriza-se por um acompanhamento diretivo, com o treinamento de habilidades de vida cotidiana, ocupacionais, sociais e de lazer, concretizado em visitas domiciliares numa busca ativa do paciente na comunidade). A equipe multidisciplinar que dá sustentação ao acompanhamento dos pacientes no PACT presta os serviços de que ele necessita, prescindindo dos serviços comunitários da rede institucionalizada ${ }^{12}$.

\section{Critical Time Intervention}

A Intervenção no Período Crítico (Critical Time Intervention - CTI) foi criada com o objetivo de complementar e fortalecer a rede de serviços de saúde e de suporte social, auxiliando pessoas portadoras de Transtornos do Espectro Esquizofrênico a se engajarem e se estabilizarem em um cuidado continuado. Trata-se de um modelo inovador, desenvolvido inicialmente para ser administrado durante o período de transição de situações críticas - como hospitalização, encarceramento, situação de rua/albergamento e ausência ou precariedade de tratamento - visando à inserção em serviços de apoio e tratamento de longo prazo $^{13}$. O modelo original foi desenvolvido para responder às condições dos servi- ços disponíveis para pessoas em situação de rua com transtornos mentais na cidade de Nova Iorque, mas versões adaptadas da CTI estão atualmente sendo testadas em outras cidades dos EUA e da Europa.

Ao contrário do Case Management e do PACT, a CTI é uma intervenção com duração de tempo pré-determinada (em geral nove meses) constituída por dois componentes. O primeiro visa o estabelecimento de vínculos estáveis dos portadores de transtornos mentais severos e persistentes com os sistemas de apoio formal e informal, principalmente com as redes de saúde e suporte social e familiar, e o segundo centra-se no apoio emocional e prático durante o tempo crítico de transição. O trabalho concentra-se em áreas consideradas cruciais para a estabilização e a inserção social do paciente na comunidade: Tratamento Psiquiátrico e Manejo da Medicação, Tratamento de Abuso de Drogas, Treinamento de habilidades da vida diária, Prevenção e Manejo de Problemas de Moradia, Manejo de dinheiro, e é realizado por agenciadores de caso e um coordenador clínico que contam com o apoio de um psiquiatra. É executado em três fases consecutivas: inicialmente, os encontros entre agenciador $\mathrm{e}$ usuário são bastante frequentes, mas o programa é planejado de forma a que os contatos entre os pares diminuam ao longo dos meses, buscando promover a autonomia do usuário ${ }^{14}$.

Um estudo clínico randomizado comprovou a efetividade da intervenção junto a portadores de Transtornos Mentais Severos que viviam em situação de rua ${ }^{15}$. A CTI foi também associada com uma diminuição dos sintomas negativos da esquizofrenia e considerada uma intervenção com relação custo-benefício favorável ${ }^{16,17}$.

Neste artigo são apresentados resultados do estudo-piloto que visou adaptar para o contexto brasileiro a "Critical Time Intervention" (CTI), criando a Intervenção em Saúde Mental para Períodos de Transição (CTI-Br) e testar a sua viabilidade (feasibility) com pessoas portadoras de transtornos do espetro esquizofrênico em tratamento nos Centros de Atenção Psicossocial do município do Rio de Janeiro. A análise concentra-se no material obtido através das notas de campo dos profissionais de nível médio que atuaram como agenciadores de caso na Fase III da pesquisa. Na discussão empreendida, as características do programa são discutidas à luz de conceitos, continuidade de cuidados e integração com outros níveis de atenção, privilegiando o debate com atenção básica. 


\section{Metodologia}

Trata-se de um estudo-piloto de intervenção, delineado sob três eixos metodológicos: 1) Pesquisa de campo, 2) Adaptação e Implementação do CTI e 3) Formação profissional de nível médio em saúde mental. O trabalho foi operacionalizado em três fases interrelacionadas. A fase I foi eminentemente exploratória. No período de fevereiro a junho de 2009, pesquisadores realizaram entrevistas com usuários, familiares e trabalhadores e participaram da rotina de trabalho de quatro Centros de Atenção Psicossocial de uma área programática do Rio de Janeiro - AP 3, quais sejam: CAPS Clarice Lispector, Linda Batista, Fernando Diniz e Ernesto Nazareth.

De julho de 2009 a fevereiro de 2010 foi realizado o mapeamento das características sociodemográficas, clínicas, de utilização de serviços e de necessidades concretas e de saúde em uma amostra de 150 usuários com transtorno do espectro esquizofrênico, através da aplicação de entrevistas semiestruturadas (Questionário Central CTI$\mathrm{Br}$ ) e questionários e escalas estruturadas (Escala das Síndromes Positiva e Negativa-PANSS; entrevista diagnóstica-MINIPLUS e Questionário de qualidade de vida - QLS-Br).

A análise do material das entrevistas com informantes-chave e dos registros dos pesquisadores sobre a rotina dos serviços foi feito por duas pesquisadoras que não participaram da atividade de campo. Para a análise quantitativa dos dados foram utilizados testes estatísticos de associação, como o teste do qui-quadrado e o teste de Fischer para variáveis categóricas e os testes-t para variáveis contínuas, considerando nível de significância de 5\%. Foram estimados os odds ratios e seus respectivos intervalos de confiança. O programa SPSS 11.0.1 (Statistic al Package for the Social Sciences) foi utilizado para a entrada de dados e posterior análise.

$\mathrm{Na}$ fase II, foi feita a tradução da CTI e sua adaptação para a realidade sociocultural brasileira, considerando as necessidades e circunstâncias particulares da população em tratamento nos CAPS. No trabalho, realizado entre os meses de janeiro e março 2010, foram traduzidos para o português o Manual Clínico do CTI (Critical Time Intervention), o Formulário de Datas das Fases, o Formulário de Supervisão; Notas sobre o progresso da intervenção e Nota de Fechamento de Caso do CTI-Br. No processo de adaptação do instrumento foram incluídas duas áreas de intervenção que não constavam no programa original: Intervenção Familiar e Violência na comunidade.
Para a execução da intervenção, foram selecionados profissionais de nível médio oriundos do Curso de Especialização Técnica de Nível Médio em Saúde Mental da Escola Politécnica de Saúde Joaquim Venâncio (EPSJV) /Fiocruz, que desde a década de 1990 forma trabalhadores de nível médio na área de saúde mental. Seguindo a orientação do Manual Clínico do CTI, a Coordenadora geral do projeto conduziu o processo de capacitação dos profissionais para atuarem como agenciadores de caso durante a implementação da intervenção.

Na Fase III, executada no período de março a dezembro de 2010, a CTI foi implementada junto a um grupo de dezenove usuários dos quatro CAPS através do acompanhamento de agenciadores de caso que trabalharam em parceria com as equipes dos serviços, sob supervisão semanal com a coordenadora do projeto. A "Intervenção para Períodos de Transição" foi implantada em três etapas consecutivas: transição, experimentação e transferência do cuidado, tendo cada uma duração média de três meses. No início da intervenção e ao final de cada uma das etapas foram aplicados questionários de avaliação da implementação. Na primeira etapa - transição - o usuário e o agenciador da CTI formularam um plano de tratamento com duração de nove meses, focado nas áreas identificadas como cruciais para o fortalecimento da estabilidade e favorecimento do engajamento na vida na comunidade. A segunda etapa - experimentação - correspondeu à implementação do plano terapêutico traçado na fase de transição, que era reavaliado semanalmente nas reuniões de supervisão do projeto. A etapa final - transferência do cuidado objetivou promover o ajuste da relação construída entre o usuário e a rede de apoio e a saída gradual do agenciador da relação de cuidado estabelecida ao longo dos nove meses.

A partir da leitura dos Planos de tratamento e dos formulários preenchidos pelos agenciadores, buscou-se extrair as informações relevantes relacionadas aos focos da intervenção, atendendo aos critérios específicos de cada fase. Foi construída uma máscara de análise para cada paciente, objetivando organizar e sistematizar os dados obtidos durante os nove meses de agenciamento de caso - com ênfase na evolução do caso e na trajetória do usuário junto ao agenciador.

\section{Aspectos éticos}

A pesquisa, coordenada pela professora Maria Tavares Cavalcanti, foi aprovada pelo Comi- 


\section{Resultados e discussão}

Dezenove pacientes foram acompanhados ao longo de nove meses pelos agenciadores do programa. Os planos terapêuticos traçados para cada paciente concentraram-se na escolha de duas ou três áreas de intervenção (ver Quadro 1). A área de "Tratamento Psiquiátrico e Manejo da Medicação" foi escolhida como foco de intervenção para 14 dos 19 integrantes do grupo. Foram várias as justificativas para tal escolha: não fazer uso regular da medicação psiquiátrica prescrita,

não frequentar o CAPS regularmente, não estar inserido no sistema de saúde mental, apresentar alterações psiquiátricas, como alucinações auditivas e insônia. Além disso, justificou-se a indicação para pacientes que não sabiam manejar a medicação, dependendo de familiares para sua administração, que não a utilizavam por não aceitarem sua doença, não tinham apoio de seus familiares, ou não acreditavam que este fosse um recurso necessário ao tratamento. A escolha foi também justificada nos casos em que havia um histórico de diversas internações psiquiátricas.

Estudo realizado por Bandeira indicou que o uso regular de medicação, a presença de suporte social, a abstinência do álcool, a aceitação da doença e a percepção mais realista de si seriam fatores que favorecem a reinserção social e protegem pessoas com transtornos mentais contra as reinternações hospitalares. No estudo, a atitude relacionada ao uso dos remédios entre os pacientes que se mantinham estáveis na comunidade não se associou à crença nos medicamentos, e a adesão ao

Quadro 1. Pacientes acompanhados pelo programa CTI-Br segundo CAPS e área de intervenção.

\begin{tabular}{|c|c|c|c|c|c|c|c|c|}
\hline & & \multicolumn{7}{|c|}{ Áreas de intervenção } \\
\hline & & $\begin{array}{c}\text { Tratamento } \\
\text { Psiquiátrico } \\
\text { e Manejo da } \\
\text { Medicação }\end{array}$ & $\begin{array}{c}\text { Tratamento } \\
\text { de Abuso } \\
\text { de Drogas }\end{array}$ & $\begin{array}{l}\text { Treinamento } \\
\text { de habilidades } \\
\text { da vida diária }\end{array}$ & $\begin{array}{c}\text { Prevenção e } \\
\text { Manejo de } \\
\text { Problemas } \\
\text { de Moradia }\end{array}$ & $\begin{array}{l}\text { Manejo de } \\
\text { dinheiro }\end{array}$ & $\begin{array}{c}\text { Intervenção } \\
\text { Familiar }\end{array}$ & $\begin{array}{l}\text { Violência na } \\
\text { comunidade }\end{array}$ \\
\hline \multirow{5}{*}{$\begin{array}{l}\text { CAPS } \\
\text { Clarice } \\
\text { Lispector }\end{array}$} & Coutinho & $\mathrm{x}$ & & $\mathrm{x}$ & & & $\mathrm{x}$ & \\
\hline & Cesar & $\mathrm{x}$ & & & $\mathrm{x}$ & $\mathrm{x}$ & & \\
\hline & Carlos & $\mathrm{x}$ & & $\mathrm{x}$ & & $\mathrm{x}$ & & \\
\hline & Ciro & $\mathrm{x}$ & & & $\mathrm{x}$ & & & $\mathrm{x}$ \\
\hline & Cecilio & $\mathrm{x}$ & & $\mathrm{x}$ & & & $\mathrm{x}$ & \\
\hline \multirow{5}{*}{$\begin{array}{l}\text { CAPS } \\
\text { Linda } \\
\text { Batista }\end{array}$} & Luzia & $\mathrm{x}$ & & & & & $\mathrm{x}$ & \\
\hline & Leandro & $\mathrm{x}$ & & & & $\mathrm{x}$ & & \\
\hline & Lindalva & $\mathrm{x}$ & & $\mathrm{x}$ & & & & \\
\hline & Luiz & $\mathrm{x}$ & & & & & $\mathrm{x}$ & \\
\hline & Lívia & $\mathrm{x}$ & & & & & & $\mathrm{x}$ \\
\hline \multirow{5}{*}{$\begin{array}{l}\text { CAPS } \\
\text { Fernando } \\
\text { Diniz }\end{array}$} & Fabiana & $\mathrm{x}$ & & $\mathrm{x}$ & & & $\mathrm{x}$ & \\
\hline & Francisco & & & $\mathrm{x}$ & & & $\mathrm{x}$ & $\mathrm{x}$ \\
\hline & Fabrício & & & & & & $\mathrm{x}$ & $\mathrm{x}$ \\
\hline & Fernanda & & $\mathrm{x}$ & & $\mathrm{x}$ & $\mathrm{x}$ & & $\mathrm{x}$ \\
\hline & Fabíola & & & & & & $\mathrm{x}$ & \\
\hline \multirow{4}{*}{$\begin{array}{l}\text { CAPS } \\
\text { Ernesto } \\
\text { Nazareth }\end{array}$} & Élcio & $\mathrm{x}$ & $\mathrm{x}$ & & & & & $\mathrm{x}$ \\
\hline & Eliane & $\mathrm{x}$ & $\mathrm{x}$ & & & & $\mathrm{x}$ & \\
\hline & Ester & & & $\mathrm{x}$ & $\mathrm{x}$ & $\mathrm{x}$ & $\mathrm{x}$ & \\
\hline & Etelvina & $\mathrm{x}$ & & & & & $\mathrm{x}$ & \\
\hline
\end{tabular}


tratamento medicamentoso pode ter resultado do esforço de pessoas que cercavam o paciente em manter em uso dos fármacos, indicando provavelmente um melhor suporte social ${ }^{18}$.

A necessidade de suporte social para a estabilidade das pessoas com transtornos mentais na comunidade foi reconhecida pelos agenciadores, que deram destaque à área de intervenção familiar escolhendo-a como foco de intervenção para 11 dos 19 pacientes. Entre as justificativas estão a saturação do convívio devido à sobrecarga familiar e o desinteresse da família pelo membro adoecido, além do desconhecimento da família sobre a doença e suas implicações.

Com o processo da desinstitucionalização psiquiátrica, os familiares de pessoas portadoras de transtornos mentais passaram a exercer cada vez mais o papel de cuidadores. Essa mudança tem o aspecto positivo de implicar as famílias no tratamento, com repercussão direta na situação estigmatização a que eram submetidas. Contudo, ao assumirem a responsabilidade pelo cuidado do paciente, as famílias a passam sofrer com a sobrecarga do cuidado, pois muitas vezes tornamse a única fonte de suporte, sem terem sido preparadas para assumirem tal responsabilidade ${ }^{19}$.

A ausência de suporte social se traduz não só na falta de apoio de familiares, mas também na ausência de suporte por parte do sistema social mais amplo. A Área de Prevenção e Manejo de Problemas de Moradia foi escolhida como foco de intervenção em quatro casos, incluindo um paciente que se encontrava em situação de rua havia 20 anos, vivendo em condições precárias.

A inclusão da área "Violência na comunidade" na adaptação brasileira do CTI deveu-se à marcante presença da violência em nossa realidade. Seis dos dezenove pacientes tiveram este tema como foco de intervenção em seus planos terapêuticos. Muitos pacientes moram em comunidades violentas, sob influência do poder de traficantes de drogas e de milícias e, pelas condições que os diferenciam de outros moradores, ficam mais expostos a agressões físicas e verbais. Alguns pacientes apresentam também problemas relacionados ao abuso de drogas que trazem risco à sua saúde e à sua segurança na comunidade, tendo sido esta área foco de intervenção para três pacientes.

Pessoas com transtornos mentais severos e persistentes podem ter grande comprometimento de sua autonomia em decorrência de questões relacionadas a condições inerentes à doença ou à longa institucionalização. Nesse sentido, a área de Treinamento de habilidades da vida diária foi foco de intervenção para sete pacientes que tinham problemas com seu cuidado pessoal, muitas vezes comprometendo sua saúde física, ou apresentavam-se muito dependentes da companhia de um familiar. O Manejo de dinheiro foi foco de intervenção para cinco pacientes, que muitas vezes não tinham qualquer fonte de renda, ficando à mercê de donativos e até mesmo, em um caso, de renda obtida através de prostituição.

Um aspecto comum a todos os pacientes que participaram da intervenção é a constatação da situação de vulnerabilidade que estavam submetidos. Para Ayres et al. a vulnerabilidade é resultante da interação de três dimensões: 1) Dimensão individual: condições de existência-materiais, corporais e psíquicas; comportamento; atitudes; escolaridade; entre outras; 2) Dimensão social (estrutura familiar; rede social; acesso à saúde, educação, seguridade social, moradia, cultura e lazer; direitos; estigma e 3) Dimensão programática/institucional (capazes de reproduzir os problemas de saúde): problemas estruturais dos serviços; problemas de gestão e financiamento; falta de articulação de rede de serviços de saúde e suporte social; barreiras de acesso aos serviços; integralidade da atenção; intersetorialidade; sustentabilidade política e institucional do sistema de saúde, etc. $)^{20}$.

A maior parte dos pacientes vivia em um ambiente extremamente pobre, sem acesso a espaços de lazer ou outras possibilidades de vínculos sociais. Os pacientes mantinham vínculos somente com seus familiares e com as equipes dos CAPS, e em nenhum dos casos pode-se contar com a ajuda de agentes comunitários de saúde ou equipes da saúde da família, inexistentes em seus territórios.

Os planos de tratamento foram traçados para que os agenciadores se aliassem aos usuários, suas famílias e às equipes dos CAPS no enfrentamento das condições que, em linhas gerais, determinavam a manutenção desta vulnerabilidade. Os agenciadores acompanharam os pacientes em seus locais de moradia, conviveram com seus familiares, estabeleceram relações com a vizinhança, os visitaram durante períodos de internação, percorreram a rede de serviço social para providenciar documentos de identidade civil e obtenção de benefícios sociais, acompanharam consultas médicas buscando a resolução de outros problemas de saúde, visando, de uma maneira ampla, a superação de barreiras que pudessem impedir a melhora do paciente e sua inserção na comunidade.

O trabalho minucioso de capacitação e supervisão dos agenciadores para a construção e a 
condução dos casos remete-nos às "ferramentas tecnológicas," utilizadas na implantação de uma rede de cuidados que integra a atenção básica aos outros níveis de atenção em saúde: apoio matricial, clínica ampliada, projeto terapêutico singular (PTS) e projeto de saúde no território (PST). O apoio matricial é realizado através de uma parceria entre a equipe de saúde da família e a equipe especializada. A Clínica Ampliada caracteriza-se pela busca de uma compreensão do processo saúde-doença que comporta diferentes matrizes teóricas para a explicação dos fenômenos envolvidos no adoecimento - seja no âmbito da biologia, da subjetividade ou das forças sociais - reconhecendo que uma apresentação clínica pode ter vários determinantes. O Projeto Terapêutico Singular (PTS) e o Projeto de Saúde no Território (PST) são estratégias de intervenção em nível individual e no território de abrangên$\mathrm{cia}^{21-23}$. Este processo de trabalho guarda semelhança com a metodologia de implantação da CTI, remetendo nossa discussão às questões próprias da atenção básica. Assim como os agenciadores da CTI, as equipes da Atenção básica se deparam com situações de risco que demandam intervenção urgente, e pela complexidade dessas situações clínicas e psicossociais os profissionais precisam de suporte técnico para sua atuação. Em estudo realizado em São Paulo, trabalhadores de saúde mental apontam que as equipes de saúde da família encaminham os casos que envolvem sofrimento psíquico para os serviços de saúde mental por não se sentirem qualificadas para interagir com experiências que estão fora do seu campo estrito de saber. Por outro lado, as equipes de saúde da família admitem que a resolução dos problemas que ali se apresentam é maior quando sua atuação vai além das condutas médicas clássicas (ou restritas a intervenções medicamentosas). Contudo, se consideram despreparadas para manejar as situações que implicam sofrimento mental ${ }^{24}$.

Avalia-se que o sucesso alcançado na adaptação e na implementação do CTI no contexto brasileiro relaciona-se ao processo de trabalho instituído, no qual o agenciador ocupa um papel central. Influiu no bom resultado da intervenção o pequeno número de casos acompanhados por cada agenciador e o contato estabelecido por estes profissionais com os CAPS e outros locais de tratamento, que incluía a participação do agenciador nas reuniões de equipe do serviço. Estudo realizado no Rio de Janeiro ${ }^{25}$ identificou que um dos principais desafios enfrentados pelas equipes dos CAPS é conciliar o cuidado "intra - CAPS" com o trabalho de acompanhamento dos seus usuários no território. A diversidade de atribuições dos CAPS, as dificuldades inerentes aos transtornos mentais, além do convívio diário das pessoas com problemas urbanos - violência, uso de drogas, desemprego - são, possivelmente, obstáculos concretos para o acesso à saúde e para a continuidade do cuidado às pessoas com transtornos mentais graves no contexto dos serviços comunitários de saúde mental. E nesse sentido, os agenciadores, ao se aliarem às equipes dos CAPS, constituíram um reforço importante no suporte daqueles pacientes.

Destaca-se, também, a importância do acompanhamento do trabalho destes agentes pelo profissional especializado: ao longo de todo o processo foram realizadas reuniões semanais com os agenciadores, nas quais o material anotado no formulário "notas do progresso da intervenção" era discutido com o supervisor. Além disso, os agenciadores tiveram acesso imediato ao supervisor sempre que se fez necessária a resolução de dificuldades surgidas na condução dos casos. A inovação das práticas em saúde mental cria uma diversidade de estratégias de cuidado e organização de serviços. A participação do agenciador na implementação da CTI reforça a importância da discussão sobre a inserção do trabalhador de nível médio na condução de práticas que contribuem para o avanço da Reforma psiquiátrica brasileira.

\section{Colaboradores}

MCA Carvalho trabalhou na análise dos dados, concepção e redação do artigo; MT Cavalcanti trabalhou na pesquisa, metodologia, análise dos dados e redação do artigo; CM Dahl, E Valencia e FM Souza trabalharam na pesquisa, metodologia e análise dos dados.

\section{Agradecimentos}

Os autores agradecem o apoio do Conselho Nacional de Desenvolvimento Científico e Tecnológico $(\mathrm{CNPq})$ ao desenvolvimento da pesquisa. 


\section{Referências}

1. Brasil. Ministério da Saúde (MS). Secretaria de Atenção à Saúde. DAPES. Coordenação Geral de Saúde Mental, Álcool e Outras Drogas. Saúde Mental no SUS: as novas fronteiras da Reforma Psiquiátrica. Relatório de Gestão 2007-2010. Brasília: Ministério da Saúde (MS); 2011.

2. Leal EM, Delgado PGG. Clínica e cotidiano: o CAPS como dispositivo de desinstitucionalização. In: Pinheiro R, Guljor AP, Gomes A, Mattos RM, organizadores. Desinstitucionalização na saúde mental: contribuições para estudos avaliativos. Rio de Janeiro: Cepesc, IMS/LAPPIS, Abrasco; 2007. p. 137-154.

3. World Health Organization (WHO). Improving Health Systems and Services for Mental Health, (Mental health policy and service guidance package). Geneva: World Health Organization (WHO); 2009.

4. Travassos C, Martins M. Uma revisão sobre os conceitos de acesso e utilização de serviços de saúde. Cad Saude Publica 2004; 20(Supl. 2):190-198.

5. Haggery JL, Reid RJ, Freeman GK, Starfield BH, Adair CE, McKendry R. Continuity of care: a multidisciplinary review. BMJ 2003; 327(7425):1219-1221.

6. Canadian Health Services Research Foundation. [acessado 2010 fev 25]. Disponível em: http://www. chsrf.cal

7. Adair C, McDougall GM, Beckie A, Joyce A, Mitton C, Wild CT, Gordon A, Costigan N. History and Measurement of Continuity of Care in Mental Health Services and Evidence of Its Role in Outcomes. Psychiatr Serv 2003; 54(10):1351-1356.

8. Johnson S, Prosser D, Bindman J, Szmukler G. Continuity of care for the severely mentally ill: concepts and measures. Soc Psychiatr Epidemiol 1997; 32(3):137-142.

9. Bachrach LL. The Challenge of Service Planning for Chronic Mental Patients. Community Ment Health J 1986; 22(3):170-174.

10. Haggery JL, Reid RJ, Freeman GK, Starfield BH, Adair CE, McKendry R. Continuity of care: a multidisciplinary review. BMJ 2003; 327(7425):12191221.

11. Adair CE, McDougall GM, Mitton CR, Joyce AS, Wild TC, Gordon A, Costigan N, Kowalsky L, Pasmeny G, Beckie A. Continuity of care and health outcomes among persons with severe mental illness. Psychiatr Serv 2005; 56(9):1061-1069.

12. Bandeira M, Gelinas D, Lesage A. Desinstitucionalização: o programa de acompanhamento intensivo na comunidade. J. Bras Psiq 1998; 47(12):627-640.

13. Valencia E, Susser E, McQuistion H, Vaccaro JV, Clark Junior GH. Critical time points in the clinical care of homeless mentally ill individuals. In: Vaccaro J, Clark G, editors. Practicing psychiatry in the community: A manual. Washington DC: American Psychiatric Press; 1996. p. 259-276.

14. Valencia E, Susser E, Torres J, Felix A, Conover S. Critical Time Intervention for Homeless Mentally ill Individuals in Transition from Shelter to Community Living. In: Breakey W, Thompson J, editors. Mentally Ill and Homeless: Special Programs for Special Needs. Amsterdam: Harwood Academic Publishers; 1997. p. 75-94.
15. Susser E, Valencia E, Conover S, Felix A, Tsai WY, Wyatt RJ. Preventing recurrent homelessness among mentally ill men: A "critical time" intervention after discharge from a shelter. Am J Public Health 1997; 87(2):256-262.

16. Herman D, Opler L, Felix A, Valencia E, Wyatt RJ, Susser E. Critical time intervention: Impact on psychiatric symptoms. J Nerv Ment Dis 2000; 188(3): $135-140$.

17. Jones K, Colson PW, Holter MC, Lin S, Valencia E, Susser E, Wyatt RJ. Costeffectiveness of critical time intervention to reduce homelessness among persons with mental illness. Psychiatric Services 2003; 54(6):884-890.

18. Bandeira M. Reinserção dos doentes mentais na comunidade: fatores determinantes das reospitalizações. J Bras Psiquiatr 1993; 42(9):491-498.

19. Bandeira M, Barroso SM. Sobrecarga das famílias de pacientes psiquiátricos. J Bras Psiquiatr 2005; 54(1):34-46.

20. Ayres JRCM, Calazans GJ, Saletti Filho HC, França-Júnior I. Risco, vulnerabilidade e práticas de prevenção e promoção da saúde. In: Campos GWS, Minayo MCS, Akerman M, Drumond Júnior M, Carvalho YM, organizadores. Tratado de Saúde Coletiva. Rio de Janeiro: Fiocruz; 2009. p. 375-419.

21. Brasil. Ministério da Saúde (MS). Secretaria de atenção à saúde. Departamento de atenção básica. $D i$ retrizes do Nasf. Núcleo de Apoio a Saúde da Família, Cadernos de Atenção básica 27. Brasília: Ministério da Saúde (MS); 2009.

22. Campos GWS, Amaral MA. A clínica ampliada e compartilhada, a gestão democrática e redes de atenção como referenciais teórico-operacionais para a reforma do hospital. Cien Saude Colet 2007; 12(4):849-859.

23. Campos GWS, Domitti AC. Apoio matricial e equipe de referência: uma metodologia para gestão do trabalho interdisciplinar em saúde Cad Saude Publica 2007; 23(2):399-407.

24. Figueiredo MD, Onocko Campos R. Saúde Mental na atenção básica à saúde de Campinas, SP: uma rede ou um emaranhado? Cien Saude Colet 2009; 14(1):129-138.

25. Cavalcanti MT, Dahl CM, Carvalho MCA, Valencia E. Critérios de admissão e continuidade de cuidados em centros de atenção psicossocial do Rio de Janeiro, RJ. Rev Saude Publica 2009; 43(Supl. 1):23-28.

Apresentado em 08/09/2011

Aprovado em 30/09/2011

Versão final apresentada em 05/10/2011 